\title{
New delay-dependent observer-based control for uncertain stochastic time-delay systems
}

\author{
Xiu-feng Miao ${ }^{1 *}$ (D) and Long-suo Li $^{2}$
}

"Correspondence: mxfaz@126.com

'School of Basic Science, Harbin University of Commerce, Harbin, China

Full list of author information is available at the end of the article

\section{Springer}

\begin{abstract}
This paper considers the problem of estimating the state vector of uncertain stochastic time-delay systems, while the system states are unmeasured. The system under study involves parameter uncertainties, noise disturbances and time delay, and they are dependent on the state. Based on the Lyapunov-Krasovskii functional approach, we present a delay-dependent condition for the existence of a state observer in terms of a linear matrix inequality. A numerical example is exploited to show the validity of the results obtained.
\end{abstract}

Keywords: Stochastic system; State estimation; Asymptotical stability; Time delay; LMI

\section{Introduction}

Uncertain stochastic time-delay systems have come to play an important role in many branches of science and engineering applications. Some recent improved research results pertaining to the analysis for stochastic time-delay systems have been reported; see [16] and the references therein. It is well known that the dynamic behaviour of many industrial processes contains inherent time delays due to the distributed nature of the systems [7-17]. Both time delay and uncertainties are inevitable in real applications. Systems have difficulty keeping nice performances, because time delay and/or uncertainties often destroy the stability of systems. As regards being based on the size of the time delays of the systems, the criteria for time-delay systems can be classified into two groups: delay-independent criteria and delay-dependent criteria. Generally speaking, since delaydependent conditions make use of information on the length of the delay, they are less conservative than delay-independent ones. To obtain delay-dependent conditions, the main approaches consist of model transformations of an original system and the bounding technique $[18,19]$.

On the other hand, the availability for direct measurements of all the state variables is rare in practice. In most cases, we need to estimate unmeasurable state variables. For this particular task, a state observer is more common, in order to accurately reconstruct the state variables of the systems [20-24]. The problem of observer design for uncertain stochastic time-delay systems has been investigated by many researchers. For example,

(c) The Author(s) 2019. This article is distributed under the terms of the Creative Commons Attribution 4.0 International License (http://creativecommons.org/licenses/by/4.0/), which permits unrestricted use, distribution, and reproduction in any medium, provided you give appropriate credit to the original author(s) and the source, provide a link to the Creative Commons license, and indicate if changes were made. 
the authors in [25] designed an exponent filter estimate state for stochastic nonlinear systems with time delay by using a high-gain observer-based approach. The authors of [26] investigated a stochastic system with process noises and sensor noises, and they gave a design method of observer minimizing the upper bound of an error variance. In [27], the authors dealt with the output feedback sliding mode control for Itô stochastic time-delay systems. The delay-independent sufficient condition for the asymptotic stability in probability of the overall closed-loop stochastic system was derived. To the best of the author's knowledge, so far little work is available in the literature that addresses a delay-dependent condition of the existence of observer for uncertain stochastic time-delay systems.

This article considers state estimation for a class of Itô-type stochastic systems subject to time delay and parameter uncertainties. The system states are unmeasured. The stochastic system involves parameter uncertainties and time delay, and they are dependent on the state. The objective is to design a robust observer such that the dynamics of the estimation error is guaranteed to be asymptotically stable in the mean square. Attention is focused on the design of the gain matrix and the state-feedback controller. This paper derived an observer design method of uncertain stochastic time-delay systems by constructing a proper Lyapunov-Krasovskii functional and by making use of the free weighting matrix method. In [27], the authors obtained some theorems, but conclusions are independent of time delay. Consequently, this will largely restrict the applying area of the conclusions. The innovation of this paper is that the delay-dependent sufficient condition for the existence of such a state observer for any admissible uncertainties is given. We present a new method to estimate the stochastic systems. Based on the new criterion, a delay-dependent condition for the existence of state observer is derived in terms of a linear matrix inequality (LMI), therefore it is in the sense of being conservative reduced. A numerical example is exploited to show the validity of the results obtained.

In this paper, we work on the complete probability space $(\Omega, \mathcal{F}, \mathcal{P})$ with the filtration $\mathcal{F}_{t\{t \geq 0\}}$ satisfying the usual conditions. $\mathbb{R}^{n}$ and $\mathbb{R}^{m \times n}$ denote, respectively, the $n$ dimensional Euclidean space and the set of all $m \times n$ real matrices. $L_{2}[0, \infty)$ is the space of square-integrable vector functions over $[0, \infty) . C^{2,1}\left(\mathbb{R}^{n} \times \mathbb{R}_{+} ; \mathbb{R}_{+}\right)$denotes the family of all nonnegative functions $V(x(t), t)$ on $\mathbb{R}^{n} \times \mathbb{R}_{+}$that are continuously twice differentiable in $x$ and once differentiable in $t$. Let $\tau>0$ and denote by $C\left([-\tau, 0] ; \mathbb{R}^{n}\right)$ the family of continuous functions $\varphi$ from $[-\tau, 0]$ to $\mathbb{R}^{n}$ with the norm $\|\varphi\|=\sup _{\theta \in[-\tau, 0]}|\varphi(\theta)|$, where $|\cdot|$ and $\|\cdot\|$ are the usual Euclidean norm in $\mathbb{R}^{n}$ and the $L_{2}[0, \infty)$ norm, respectively. $P>0$ means the matrix $P$ is symmetric positive definite, the notation $U>V$, where $U$ and $V$ are symmetric matrices, means that $U-V$ is a positive definite matrix. $\mathbb{E}(x)$ stands for the expectation of stochastic variable $x, B^{T}$ represents the transposed matrix of $B, I$ denote the identity matrix of compatible dimension; moreover, $\left[\begin{array}{ll}A & B \\ * & D\end{array}\right]=\left[\begin{array}{cc}A & B \\ B^{T} & B\end{array}\right]$. The shorthand $\operatorname{diag}\left\{F_{1}, \ldots, F_{n}\right\}$ denotes a block diagonal matrix with diagonal blocks being the matrices $F_{1}, \ldots, F_{n}$.

\section{Preliminaries}

Consider the following stochastic time-delay systems described in Itô's form:

$$
\begin{aligned}
& \mathrm{d} x(t)=\left[(A+\Delta A(t)) x(t)+\left(A_{\tau}+\Delta A_{\tau}(t)\right) x(t-\tau)+B u(t)\right] \mathrm{d} t+D x(t) \mathrm{d} \omega(t), \\
& x(t)=\varphi(t), \quad t \in[-\tau, 0], \\
& y(t)=C x(t),
\end{aligned}
$$


where $x(t) \in \mathbb{R}^{n}$ is the state, $u(t) \in \mathbb{R}^{p}$ is the control input, $y(t) \in \mathbb{R}^{r}$ is the measured output, $\tau$ is a real constant time delay satisfying $0 \leq \tau<\infty . \varphi(t) \in C\left([-\tau, 0]\right.$; $\left.\mathbb{R}^{n}\right)$ is a continuous vector-valued initial function, and $\omega(t)$ is a one-dimensional Brownian motion satisfying

$$
\mathbb{E}[\mathrm{d} \omega(t)]=0, \quad \mathbb{E}\left[\mathrm{d} \omega^{2}(t)\right]=\mathrm{d}(t) .
$$

Here, $A \in \mathbb{R}^{n \times n}, A_{\tau} \in \mathbb{R}^{n \times n}, B \in \mathbb{R}^{n \times p}, D \in \mathbb{R}^{n \times n}, C \in \mathbb{R}^{r \times n}$ are known real constant matrices of appropriate dimensions. Moreover, $\Delta A(t)$ and $\Delta A_{\tau}(t)$ are unknown matrices representing time-varying parameter uncertainties, and they are assumed to be of the form

$$
\left[\Delta A(t) \quad \Delta A_{\tau}(t)\right]=G F(t)\left[\begin{array}{ll}
H_{1} & H_{2}
\end{array}\right]
$$

where $G, H_{1}, H_{2}$ are known real constant matrices and $F(t)$ is an unknown time-varying matrix function satisfying

$$
F(t)^{T} F(t) \leq I, \quad \forall t
$$

The parameter uncertainties $\Delta A(t)$ and $\Delta A_{\tau}(t)$ are said to be admissible if both (2) and (3) hold.

Definition 1 ([28]) System (1a)-(1c) is said to be robustly asymptotically mean-square stable if for all admissible uncertainties (2) and (3) the following holds for any initial condition:

$$
\lim _{t \rightarrow \infty} \mathbb{E}\left\{\|x(t)\|^{2}\right\}=0
$$

Remark 1 Uncertain stochastic time-delay systems in the form of (1a)-(1c) are common in many branches of engineering applications $[1,3,5]$. It is observed that, in system (1a)(1c), parameter uncertainties, Itô-type stochastic disturbances and time delay are considered simultaneously, also they are dependent on the state. The aim of this paper is to design a state observer of system (1a)-(1c) such that dynamics of the estimation error is asymptotically stable in the mean square.

Before presenting the main results of this article, we first introduce the following several lemmas, which will be essential for later developments.

Lemma 1 (Schur complement [29]) For a given the symmetric matrix $X=\left[\begin{array}{ll}X_{11} & X_{12} \\ X_{12}^{T} & X_{22}\end{array}\right]$, the following conditions are equivalent:

(a) $X<0$,

(b) $X_{11}<0, X_{22}-X_{12}^{T} X_{11}^{-1} X_{12}<0$,

(c) $X_{22}<0, X_{11}-X_{12} X_{22}^{-1} X_{12}^{T}<0$.

Lemma 2 ([27]) Let $G, H$ and $F(t)$ be real matrices of appropriate dimensions with $F(t)$ satisfying $F(t)^{T} F(t) \leq I$. Then, for any scalar $\varepsilon>0$, we have

$$
G F(t) H+H^{T} F(t)^{T} G^{T} \leq \varepsilon G G^{T}+\varepsilon^{-1} H^{T} H .
$$


Lemma 3 ([30]) Let us have any positive definite matrix $R>0$, scalar $\tau>0$. If there exists a vector function $x(t):[0, \tau] \rightarrow \mathbb{R}^{n}$, such that $\int_{0}^{\tau} x^{T}(s) R x(s) \mathrm{d} s$ and $\int_{0}^{\tau} x^{T}(s) \mathrm{d} s$ are well defined, then we have the following inequality:

$$
-\tau \int_{0}^{\tau} x^{T}(s) R x(s) \mathrm{d} s \leq-\int_{0}^{\tau} x^{T}(s) \mathrm{d} s \cdot R \cdot \int_{0}^{\tau} x(s) \mathrm{d} s .
$$

\section{Main results}

We design an observer to asymptotically estimate $x(t)$. Let us propose the following Luenberger-type observer [31] of the uncertain stochastic time-delay system (1a)-(1c):

$$
\mathrm{d} \hat{x}(t)=\left[A \hat{x}(t)+A_{\tau} \hat{x}(t-\tau)+B u(t)+L(y(t)-C \hat{x}(t))\right] \mathrm{d} t,
$$

where $L \in \mathbb{R}^{n \times r}$ is the observer gain to be designed later. From (1a)-(1c) and (4), the error vector $e(t)=x(t)-\hat{x}(t)$ can be expressed as

$$
\begin{aligned}
\mathrm{d} e(t)= & {\left[(A-L C+\Delta A(t)) e(t)+\left(A_{\tau}+\Delta A_{\tau}(t)\right) e(t-\tau)+\Delta A(t) \hat{x}(t)\right.} \\
& \left.+\Delta A_{\tau}(t) \hat{x}(t-\tau)\right] \mathrm{d} t+(D \hat{x}(t)+D e(t)) \mathrm{d} \omega(t) .
\end{aligned}
$$

We introduce the following new state variable for convenience:

$$
\begin{aligned}
\bar{x}(t)= & A \hat{x}(t)+A_{\tau} \hat{x}(t-\tau)+B u(t)+L(y(t)-C \hat{x}(t)), \\
\bar{e}(t)= & (A-L C+\Delta A(t)) e(t)+\left(A_{\tau}+\Delta A_{\tau}(t)\right) e(t-\tau)+\Delta A(t) \hat{x}(t) \\
& +\Delta A_{\tau}(t) \hat{x}(t-\tau),
\end{aligned}
$$

then we rewrite the systems (4) and (5) as

$$
\mathrm{d} \hat{x}(t)=\bar{x}(t) \mathrm{d} t, \quad \mathrm{~d} e(t)=\bar{e}(t) \mathrm{d} t+(D \hat{x}(t)+D e(t)) \mathrm{d} \omega(t) .
$$

Next, we will analyze the stability of the observer system (4) and the error system (5). We aim at designing a gain matrix and a state-feedback matrix such that the error systems are asymptotically stable in the mean square. In the following theorem, we present a delaydependent LMI condition for the observer design of the error systems with $u(t) \equiv 0$.

Theorem 1 Consider the stochastic time-delay system (1a)-(1c) with $u(t) \equiv 0$. The state observer has the form of (4). If there exist matrices $P>0, Q_{1}>0, Q_{2}>0, N_{1}, N_{2}, N_{3}, N_{4}$, and positive scalar $\varepsilon>0$ satisfying the following linear matrix inequality $(L M I)$ :

$$
\left[\begin{array}{cccccccccc}
(1,1) & (1,2) & (1,3) & 0 & -N_{1} & 0 & A^{T} P & 0 & 0 & 0 \\
* & (2,2) & 0 & (2,4) & 0 & -N_{3} & C^{T} Y^{T} & (2,8) & P G & 0 \\
* & * & (3,3) & 0 & -N_{2} & 0 & A_{\tau}^{T} P & 0 & 0 & 0 \\
* & * & * & (4,4) & 0 & -N_{4} & 0 & A_{\tau}^{T} P & 0 & 0 \\
* & * & * & * & -\tau^{-1} P & 0 & 0 & 0 & 0 & 0 \\
* & * & * & * & * & -\tau^{-1} P & 0 & 0 & 0 & 0 \\
* & * & * & * & * & * & -\tau^{-1} P & 0 & 0 & 0 \\
* & * & * & * & * & * & * & -\tau^{-1} P & 0 & P G \\
* & * & * & * & * & * & * & * & \frac{1}{4} \varepsilon I & 0 \\
* & * & * & * & * & * & * & * & * & \frac{1}{4} \varepsilon I
\end{array}\right]<0, \quad(9)
$$


with

$$
\begin{aligned}
& (1,1)=A^{T} P+P A+Q_{1}+D^{T} P D+2 \varepsilon H_{1}^{T} H_{1}+N_{1}, \quad(1,2)=Y C+D^{T} P D, \\
& (1,3)=P A_{\tau}-N_{1}+N_{2}^{T}, \quad(2,4)=P A_{\tau}-N_{3}+N_{4}^{T}, \\
& (2,2)=A^{T} P+P A-Y C-C^{T} Y^{T}+Q_{2}+D^{T} P D+2 \varepsilon H_{1}^{T} H_{1}+N_{3}, \\
& (3,3)=2 \varepsilon H_{2}^{T} H_{2}-Q_{1}-N_{2}, \quad(4,4)=2 \varepsilon H_{2}^{T} H_{2}-Q_{2}-N_{4}, \\
& (2,8)=A^{T} P-C^{T} Y^{T},
\end{aligned}
$$

then the error systems are asymptotically stable in the mean square, and the observer gain is given by $L=P^{-1} Y$.

Proof Let $\bar{P}=\left[\begin{array}{ll}P & 0 \\ 0 & P\end{array}\right]>0, \bar{Q}=\left[\begin{array}{cc}Q_{1} & 0 \\ 0 & Q_{2}\end{array}\right]>0$. Now we choose a Lyapunov-Krasovskii functional candidate $V(\hat{x}(t), e(t)) \in C^{2,1}\left(\mathbb{R}^{n} \times \mathbb{R}_{+} ; \mathbb{R}_{+}\right)$as follows:

$$
\begin{aligned}
& V(\hat{x}(t), e(t), t)=\left[\begin{array}{ll}
x^{T}(t) & e^{T}(t)
\end{array}\right] \bar{P}\left[\begin{array}{ll}
x^{T}(t) & e^{T}(t)
\end{array}\right]^{T} \\
& +\int_{t-\tau}^{t}\left[x^{T}(t) \quad e^{T}(t)\right] \bar{Q}\left[x^{T}(t) \quad e^{T}(t)\right]^{T} \mathrm{~d} s \\
& +\int_{t-\tau}^{t} \int_{s}^{t} \bar{x}^{T}(\alpha) P \bar{x}(\alpha) \mathrm{d} \alpha \mathrm{d} s+\int_{t-\tau}^{t} \int_{s}^{t} \bar{e}^{T}(\alpha) P \bar{e}(\alpha) \mathrm{d} \alpha \mathrm{d} s .
\end{aligned}
$$

By using Itô's formula [32], we obtain the differential operator

$$
\begin{aligned}
& \mathcal{L} V(\hat{x}(t), e(t), t) \\
&=2 \hat{x}^{T}(t) P \bar{x}(t)+2 e^{T}(t) P \bar{e}(t)+[D \hat{x}(t)+D e(t)]^{T} P[D \hat{x}(t)+D e(t)] \\
&+\hat{x}^{T}(t) Q_{1} \hat{x}(t)-\hat{x}^{T}(t-\tau) Q_{1} \hat{x}(t-\tau)+e^{T}(t) Q_{1} e(t)-e^{T}(t-\tau) Q_{1} e(t-\tau) \\
&+\tau \bar{x}^{T}(t) P \bar{x}(t)-\int_{t-\tau}^{t} \bar{x}^{T}(s) P \bar{x}(s) \mathrm{d} s+\tau \bar{e}^{T}(t) P \bar{e}(t)-\int_{t-\tau}^{t} \bar{e}^{T}(s) P \bar{e}(s) \mathrm{d} s .
\end{aligned}
$$

By using the well-known Leibniz-Newton formula inequality, we have

$$
\begin{aligned}
& \mathbb{E}\left[\left(\hat{x}^{T}(t) N_{1}+\hat{x}^{T}(t-\tau) N_{2}\right)\left(\hat{x}(t)-\hat{x}(t-\tau)-\int_{t-\tau}^{t} \hat{x}(s) \mathrm{d} s\right)\right]=0, \\
& \mathbb{E}\left[\left(e^{T}(t) N_{3}+e^{T}(t-\tau) N_{4}\right)\left(e(t)-e(t-\tau)-\int_{t-\tau}^{t} e(s) \mathrm{d} s\right)\right]=0 .
\end{aligned}
$$

Using the properties of stochastic integral, one has

$$
\mathbb{E}\left[\left(e^{T}(t) N_{3}+e^{T}(t-\tau) N_{4}\right)\left(\int_{t-\tau}^{t}(D \hat{x}(t)+D e(t)) \mathrm{d} \omega(t)\right)\right]=0 .
$$

Noticing Eq. (8), one has

$$
\mathbb{E}\left[\left(\hat{x}^{T}(t) N_{1}+\hat{x}^{T}(t-\tau) N_{2}\right)\left(\hat{x}(t)-\hat{x}(t-\tau)-\int_{t-\tau}^{t} \bar{x}(s) \mathrm{d} s\right)\right]=0,
$$




$$
\mathbb{E}\left[\left(e^{T}(t) N_{3}+e^{T}(t-\tau) N_{4}\right)\left(e(t)-e(t-\tau)-\int_{t-\tau}^{t} \bar{e}(s) \mathrm{d} s\right)\right]=0
$$

Furthermore, we can also obtain the following two inequalities from Lemma 3:

$$
\begin{aligned}
& -\tau \int_{t-\tau}^{t} \bar{x}^{T}(s) P \bar{x}(s) \mathrm{d} s \leq\left(\int_{t-\tau}^{t} \bar{x}^{T}(s) \mathrm{d} s\right) \cdot(-P) \cdot\left(\int_{t-\tau}^{t} \bar{x}(s) \mathrm{d} s\right), \\
& -\tau \int_{t-\tau}^{t} \bar{e}^{T}(s) P \bar{e}(s) \mathrm{d} s \leq\left(\int_{t-\tau}^{t} \bar{e}^{T}(s) \mathrm{d} s\right) \cdot(-P) \cdot\left(\int_{t-\tau}^{t} \bar{e}(s) \mathrm{d} s\right) .
\end{aligned}
$$

Adding the left sides of Eqs. (12) and (13) to $\mathcal{L} V(\hat{x}(t), e(t), t)$, combining with (14) and (15), we have

$$
\mathbb{E} \mathcal{L} V(\hat{x}(t), e(t), t) \leq \mathbb{E}\left[\eta^{T}(t) \Sigma \eta(t)\right]
$$

where

$$
\begin{aligned}
& \Sigma=\left[\begin{array}{cccccc}
(1,1) & (1,2) & P A_{\tau}-N_{1}+N_{2}^{T} & 0 & -N_{1} & 0 \\
* & (2,2) & P \Delta A_{\tau}^{T}(t) & (2,4) & 0 & -N_{3} \\
* & * & -Q_{1}-N_{2} & 0 & -N_{2} & 0 \\
* & * & * & -Q_{2}-N_{4} & 0 & -N_{4} \\
* & * & * & * & -\tau^{-1} P & 0 \\
* & * & * & * & * & -\tau^{-1} P
\end{array}\right] \\
& +\left[\begin{array}{c}
A^{T} \\
C^{T} L^{T} \\
A_{\tau}^{T} \\
0 \\
0 \\
0
\end{array}\right](\tau P)\left[\begin{array}{c}
A^{T} \\
C^{T} L^{T} \\
A_{\tau}^{T} \\
0 \\
0 \\
0
\end{array}\right]^{T} \\
& +\left[\begin{array}{c}
\Delta A^{T}(t) \\
(A-L C+\Delta A(t))^{T} \\
\Delta A_{\tau}^{T}(t) \\
\left(A_{\tau}+\Delta A_{\tau}(t)\right)^{T} \\
0 \\
0
\end{array}\right](\tau P)\left[\begin{array}{c}
\Delta A^{T}(t) \\
(A-L C+\Delta A(t))^{T} \\
\Delta A_{\tau}^{T}(t) \\
\left(A_{\tau}+\Delta A_{\tau}(t)\right)^{T} \\
0 \\
0
\end{array}\right] \text {, }
\end{aligned}
$$

with

$$
\begin{aligned}
& \eta(t)=\left[\begin{array}{llllll}
\hat{x}^{T}(t) & e^{T}(t) & \hat{x}^{T}(t-\tau) & e^{T}(t-\tau) & \int_{t-\tau}^{t} \bar{x}^{T}(s) \mathrm{d} s & \int_{t-\tau}^{t} \bar{e}^{T}(s) \mathrm{d} s
\end{array}\right]^{T}, \\
& (1,1)=A^{T} P+P A+Q_{1}+D^{T} P D+N_{1}, \\
& (1,2)=\Delta A^{T}(t) P+P L C+D^{T} P D, \\
& (2,2)=(A-L C+\Delta A(t))^{T} P+P(A-L C+\Delta A(t))+Q_{2}+D^{T} P D+N_{3}, \\
& (2,4)=P\left(A_{\tau}+\Delta A_{\tau}^{T}(t)\right)-N_{3}+N_{4}^{T} .
\end{aligned}
$$


By applying the Schur decomposition result, we can see that $\Sigma<0$ if and only if

$$
\left[\begin{array}{cccccccc}
(1,1) & (1,2) & P A_{\tau}-N_{1}+N_{2}^{T} & 0 & -N_{1} & 0 & A^{T} & (1,8) \\
* & (2,2) & P \Delta A_{\tau}^{T}(t) & (2,4) & 0 & -N_{3} & C^{T} L^{T} & (2,8) \\
* & * & -Q_{1}-N_{2} & 0 & -N_{2} & 0 & A_{\tau}^{T} & (3,8) \\
* & * & * & -Q_{2}-N_{4} & 0 & -N_{4} & 0 & (4,8) \\
* & * & * & * & -\tau^{-1} P & 0 & 0 & 0 \\
* & * & * & * & * & -\tau^{-1} P & 0 & 0 \\
* & * & * & * & * & * & -\tau^{-1} P^{-1} & 0 \\
* & * & * & * & * & * & * & -\tau^{-1} P^{-1}
\end{array}\right]<0
$$

with

$$
\begin{aligned}
& (1,1)=A^{T} P+P A+Q_{1}+D^{T} P D+N_{1}, \\
& (1,2)=\Delta A^{T}(t) P+P L C+D^{T} P D \\
& (2,2)=(A-L C+\Delta A(t))^{T} P+P(A-L C+\Delta A(t))+Q_{2}+D^{T} P D+N_{3}, \\
& (2,4)=P\left(A_{\tau}+\Delta A_{\tau}^{T}(t)\right)-N_{3}+N_{4}^{T} \\
& (1,8)=\Delta A^{T}(t) \\
& (2,8)=(A-L C+\Delta A(t))^{T} \\
& (3,8)=\Delta A_{\tau}^{T}(t) \\
& (4,8)=\left(A_{\tau}+\Delta A_{\tau}(t)\right)^{T} .
\end{aligned}
$$

Noting that pre- and post-multiplying by $\operatorname{diag}\{I, I, I, I, I, I, P, P\}$ and considering the condition (2) (3), we can write the matrix inequality (18) as

$$
\left.\begin{array}{cccccccc}
(1,1) & (1,2) & P A_{\tau}-N_{1}+N_{2}^{T} & 0 & -N_{1} & 0 & A^{T} P & 0 \\
* & (2,2) & 0 & (2,4) & 0 & -N_{3} & C^{T} L^{T} P & (A-L C)^{T} P \\
* & * & -Q_{1}-N_{2} & 0 & -N_{2} & 0 & A_{\tau}^{T} P & 0 \\
* & * & * & -Q_{2}-N_{4} & 0 & -N_{4} & 0 & A_{\tau}^{T} P \\
* & * & * & * & -\tau^{-1} P & 0 & 0 & 0 \\
* & * & * & * & * & -\tau^{-1} P & 0 & 0 \\
* & * & * & * & * & * & -\tau^{-1} P & 0 \\
* & * & * & * & * & * & * & -\tau^{-1} P
\end{array}\right]
$$

with

$$
\begin{aligned}
& (1,1)=A^{T} P+P A+Q_{1}+D^{T} P D+N_{1}, \\
& (1,2)=P L C+D^{T} P D, \\
& (2,2)=(A-L C)^{T} P+P(A-L C)+Q_{2}+D^{T} P D+N_{3}, \\
& (2,4)=P A_{\tau}-N_{3}+N_{4}^{T} .
\end{aligned}
$$

Here, $\bar{H}=\left[\left(H_{1}\right)_{1,1},\left(H_{1}\right)_{2,2},\left(H_{2}\right)_{3,3},\left(H_{2}\right)_{4,4},\left(H_{1}\right)_{5,1},\left(H_{1}\right)_{6,2},\left(H_{2}\right)_{7,3},\left(H_{2}\right)_{8,4}\right]$ denotes a block square matrix whose all nonzero blocks are the 11 st block $H_{1}$, the 21 st block $H_{2}, \ldots$, the 
84th block $H_{2}$, and all other blocks are zero matrices, i.e.,

$$
\bar{H}=\left[\begin{array}{cccc}
H_{1} & 0 & 0 & 0 \\
0 & H_{1} & 0 & 0 \\
0 & 0 & H_{2} & 0 \\
0 & 0 & 0 & H_{2} \\
H_{1} & 0 & 0 & 0 \\
0 & H_{1} & 0 & 0 \\
0 & 0 & H_{2} & 0 \\
0 & 0 & 0 & H_{2}
\end{array}\right] .
$$

Similarly, $\bar{G}=\left[(P G)_{2,1},(P G)_{2,2},(P G)_{2,3},(P G)_{2,4},(P G)_{8,5},(P G)_{8,6},(P G)_{8,7},(P G)_{8,8}\right]$, furthermore, $\bar{F}(t)$ denotes a block diagonal matrix with diagonal blocks being the matrix $F(t)$.

Let $L=P^{-1} Y$. By utilizing Lemma 2 and the Schur decomposition result again, it follows that the matrix inequality (19) is implied by the LMI (9) for a scalar $\varepsilon>0$, which guarantees that $\mathbb{E} \mathcal{L} V(\hat{x}(t), e(t), t)<0$. Hence, the trivial solution of the error system is asymptotically stable in the mean square.

Remark 2 A delay-dependent sufficient condition for the existence of the observer is given in Theorem 1. It is easy to see that the LMI condition in (9) is dependent on the length of delay and is less conservative than the delay-independent ones. The largest upper bound of the delay $\tau$ such that the LMI in (9) holds can be obtained by solving the following LMI problem:

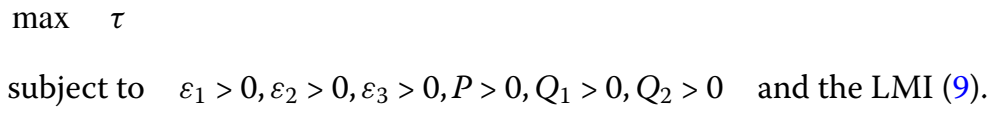

Next, we consider the state-feedback-based controller problem for the stochastic timedelay systems (1a)-(1c). A delay-dependent LMI technique will be developed in order to obtain the state-feedback observer. The following theorem shows that the controller is reachable in the stochastic theory.

Theorem 2 Consider the uncertain stochastic time-delay system (1a)-(1c). The state observer has the form of (4). If there exist matrices $P>0, Q_{1}>0, Q_{2}>0, N_{1}, N_{2}, N_{3}, N_{4}$, and positive scalars $\varepsilon>0, \sigma>0$ such that the following LMI is satisfied:

$$
\left[\begin{array}{cccccccccccc}
(1,1) & (1,2) & (1,3) & 0 & -N_{1} & 0 & A^{T} P & 0 & 0 & 0 & 0 & P B \\
* & (2,2) & 0 & (2,4) & 0 & -N_{3} & C^{T} Y^{T} & (2,8) & P G & 0 & 0 & 0 \\
* & * & (3,3) & 0 & -N_{2} & 0 & A_{\tau}^{T} P & 0 & 0 & 0 & 0 & 0 \\
* & * & * & (4,4) & 0 & -N_{4} & 0 & A_{\tau}^{T} P & 0 & 0 & 0 & 0 \\
* & * & * & * & -\tau^{-1} P & 0 & 0 & 0 & 0 & 0 & 0 & 0 \\
* & * & * & * & * & -\tau^{-1} P & 0 & 0 & 0 & 0 & 0 & 0 \\
* & * & * & * & * & * & -\tau^{-1} P & 0 & 0 & 0 & P B & 0 \\
* & * & * & * & * & * & * & -\tau^{-1} P & 0 & P G & 0 & 0 \\
* & * & * & * & * & * & * & * & \frac{1}{4} \varepsilon I & 0 & 0 & 0 \\
* & * & * & * & * & * & * & * & * & \frac{1}{4} \varepsilon I & 0 & 0 \\
* & * & * & * & * & * & * & * & * & * & \sigma I & 0 \\
* & * & * & * & * & * & * & * & * & * & * & -\frac{1}{3} \sigma I
\end{array}\right]<0,
$$


with

$$
\begin{aligned}
& (1,1)=A^{T} P+P A+Q_{1}+D^{T} P D+2 \varepsilon H_{1}^{T} H_{1}+N_{1}, \\
& (1,2)=Y C+D^{T} P D, \quad(1,3)=P A_{\tau}-N_{1}+N_{2}^{T}, \\
& (2,4)=P A_{\tau}-N_{3}+N_{4}^{T}, \\
& (2,2)=A^{T} P+P A-Y C-C^{T} Y^{T}+Q_{2}+D^{T} P D+2 \varepsilon H_{1}^{T} H_{1}+N_{3}, \\
& (3,3)=2 \varepsilon H_{2}^{T} H_{2}-Q_{1}-N_{2}, \quad(4,4)=2 \varepsilon H_{2}^{T} H_{2}-Q_{2}-N_{4}, \\
& (2,8)=A^{T} P-C^{T} Y^{T},
\end{aligned}
$$

then the overall closed-loop stochastic time-delay system is asymptotically stable in the mean square. In this case, the observer gain is given by $L=P^{-1} Y$ and an appropriate robust stabilizing state-feedback controller can be chosen as $u(x)=K \hat{x}(t), K=\sigma^{-1} B^{T} P$.

Proof Applying the controller $u(x)=\sigma^{-1} B^{T} P \hat{x}(t)$ to the system (4), and similar to the proof of Theorem 1, we can obtain $\mathbb{E} \mathcal{L} V(\hat{x}(t), e(t), t)<0$ if LMI (20) is satisfied. It implies that the trivial solution of the closed-loop system is asymptotically stable in the mean square.

Theorem 2 provides a delay-dependent sufficient condition for the existence of the robust observer of uncertain stochastic time-delay systems by state feedback. A desired gain matrix and state-feedback controller can be obtained by solving the LMI (20).

Remark 3 In [33], the state estimation method is given for a class of stochastic systems, but the stochastic disturbance is independent on the system state. However, the stochastic disturbance of uncertain systems we investigated in this paper is state dependent. In [34], the disturbance-observer design method is given for time-delay uncertain systems, and the considered uncertainties do not contain stochastic disturbances by Brown motion. The systems in this paper include not only parameter uncertainties but also a stochastic disturbance, it is derived that the closed-loop system maintains good stability despite the presence of parameter uncertainties and a stochastic factor by Brown motion. Then the method of this paper has some advantage in convergence property, the proposed method can be applied to a more general class of nonlinear systems.

\section{Simulation study}

In order to illustrate the usefulness and flexibility of the theory developed in the above section, we present a simple numerical example in this section.

Example 1 We consider the uncertain stochastic time-delay system (1a)-(1c) with

$$
\begin{aligned}
A & =\left[\begin{array}{ccc}
-1.5 & 0.2 & 0.2 \\
-1.25 & -1.3 & 1 \\
1.1 & 0.2 & -1.2
\end{array}\right], \quad A_{\tau}=\left[\begin{array}{ccc}
-1.4 & 0.5 & -0.6 \\
-0.4 & -0.2 & -1.2 \\
0.1 & 1 & -1.6
\end{array}\right], \\
D & =\left[\begin{array}{ccc}
-0.01 & 0.02 & 0.15 \\
0.12 & -0.03 & 0.02 \\
0.06 & -0.03 & 0.15
\end{array}\right], \quad B=\left[\begin{array}{lll}
0.01 & 0.02 & 0.2
\end{array}\right]^{T}, \quad C=\left[\begin{array}{lll}
1 & 1 & 0
\end{array}\right],
\end{aligned}
$$




$$
\begin{aligned}
& F(t)=0.2 \sin (t), \quad G=\left[\begin{array}{lll}
0.01 & 0.02 & 0.2
\end{array}\right]^{T}, \\
& H_{1}=\left[\begin{array}{lll}
0.03 & 0.03 & 0.01
\end{array}\right], \quad H_{2}=\left[\begin{array}{lll}
0.01 & 0.02 & 0.05
\end{array}\right] .
\end{aligned}
$$

Solving LMI (20) yields the maximum allowable bound of the time delay as $\tau=0.4454$. This means that, for any time delay $\tau$ satisfying $0<\tau \leq 0.4454$, there exist a gain matrix and a state-feedback matrix such that the resulting augmented system is asymptotically stable in the mean square. For this example, if we choose the time delay as $\tau=0.2$, then, by using the Matlab control toolbox to solve the LMI (20), we obtain

$$
\begin{aligned}
& P=\left[\begin{array}{ccc}
1.2345 & -0.3159 & -0.0001 \\
-0.3159 & 0.9683 & -0.1272 \\
-0.0001 & -0.1272 & 0.8011
\end{array}\right], \quad Q_{1}=\left[\begin{array}{ccc}
1.5954 & -0.1394 & -0.3721 \\
-0.1394 & 1.5292 & -0.7039 \\
-0.3721 & -0.7039 & 1.2157
\end{array}\right] \text {, } \\
& Q_{2}=\left[\begin{array}{ccc}
1.6596 & -0.1156 & -0.2824 \\
-0.1156 & 1.6058 & -0.6390 \\
-0.2824 & -0.6390 & 1.3066
\end{array}\right], \quad N_{1}=\left[\begin{array}{ccc}
-0.9757 & 0.2730 & -0.0499 \\
0.2730 & -0.4601 & 0.1040 \\
-0.0499 & 0.1040 & -0.9590
\end{array}\right] \text {, } \\
& N_{2}=\left[\begin{array}{ccc}
0.4629 & -0.1114 & 0.1730 \\
-0.1114 & 0.1556 & 0.1018 \\
0.1730 & 0.1018 & 0.5298
\end{array}\right], \quad N_{3}=\left[\begin{array}{ccc}
-0.9673 & 0.2660 & -0.0279 \\
0.2660 & -0.4891 & 0.0700 \\
-0.0279 & 0.0700 & -0.9134
\end{array}\right] \text {, } \\
& N_{4}=\left[\begin{array}{ccc}
0.4289 & -0.1119 & 0.1311 \\
-0.1119 & 0.1588 & 0.0585 \\
0.1311 & 0.0585 & 0.4984
\end{array}\right], \quad \varepsilon=5.7143, \quad \sigma=0.7306 \text {. }
\end{aligned}
$$

By Theorem 2, we can obtain the desired gain matrix and state-feedback matrix as follows:

$$
L=P^{-1} Y=\left[\begin{array}{lll}
0.1165 & 0.1139 & 0.1520
\end{array}\right]^{T}, \quad K=\left[\begin{array}{lll}
0.0825 & 0.1870 & 0.1845
\end{array}\right] .
$$

State response trajectories for the open-loop and closed-loop systems are given. Figure 1 is for the state response trajectories for the open-loop systems without uncertainties, Figs. 2-4 are the state response trajectories for the open-loop systems with uncertainties. We can see that the system is stable without parameter uncertainties and stochastic disturbances in Fig. 1, but from Figs. 2-4, the parameter uncertainties and stochastic disturbances makes the stability of the system decline. In [35], Higham gives a numerical

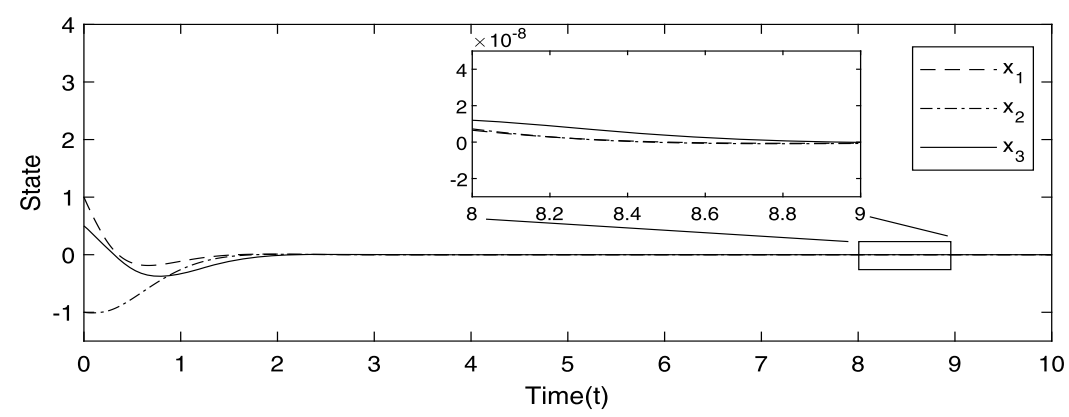

Figure 1 State response trajectories for the open-loop systems without uncertainties 


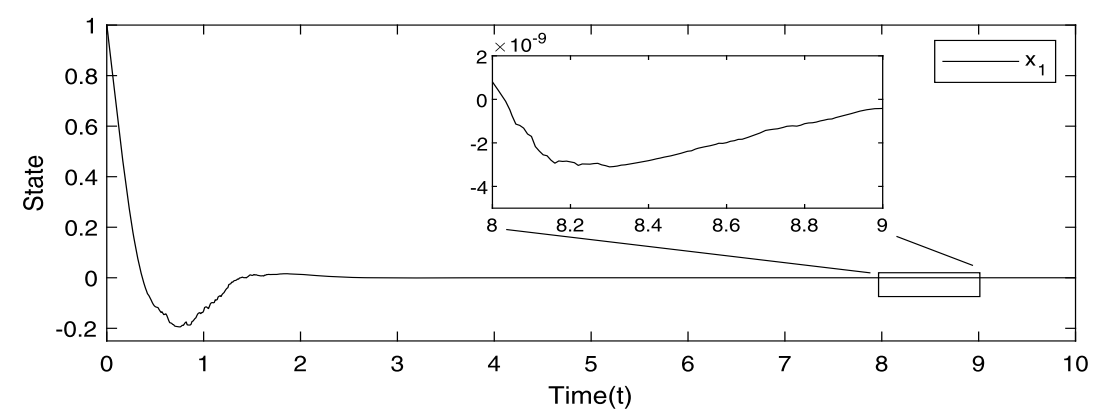

Figure 2 Response trajectory of $x_{1}$ for the open-loop systems with uncertainties

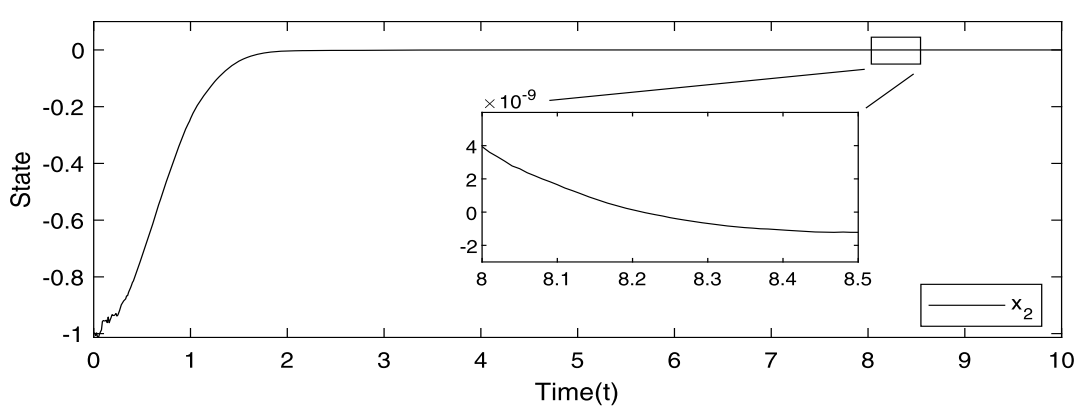

Figure 3 Response trajectory of state $x_{2}$ for the open-loop systems with uncertainties

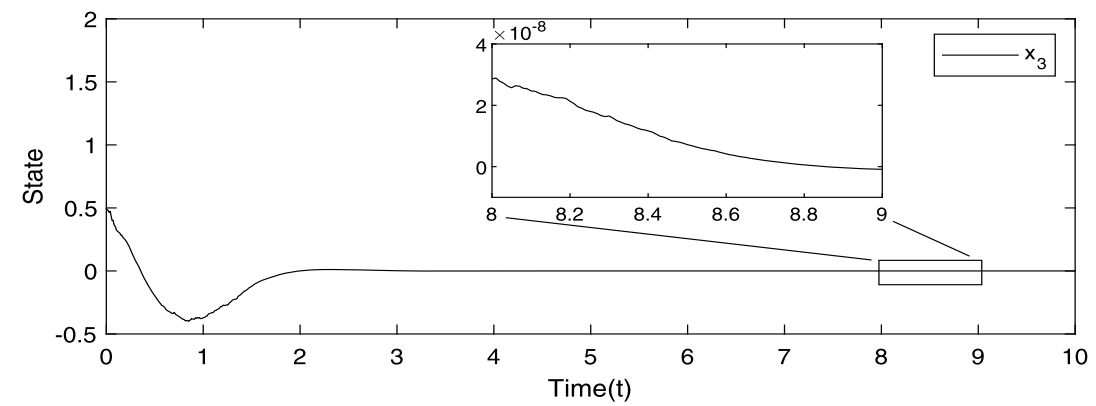

Figure 4 Response trajectory of state $x_{3}$ for the open-loop systems with uncertainties

simulation algorithm for the system with stochastic disturbances. Now, by using a similar approach, we take the initial parameters as $x(t)=\left(\begin{array}{lll}1 & -1 & 0.5\end{array}\right)^{T}, \hat{x}(t)=\left(\begin{array}{lll}-1.5 & 1 & -1.5\end{array}\right)^{T}$, $t \in[-0.2,0]$, the simulation time $t \in[0, T]$ with $T=10, \delta t=T / N$, with $N=10^{4}$, step size $\Delta t=R \delta t$ with $R=5$. Figures 5-7 show the average trajectories over eight paths of $x_{1}(t)$, $x_{2}(t)$ and $x_{3}(t)$ and their estimates, respectively. From the figures, we can see that the simulation result is satisfactory. The control performance of the state and parametric estimation is still very well despite the presence of the stochastic factor.

\section{Conclusions}

This article considers the estimation of the state vector of stochastic systems with time delay and parametrical uncertainty. The delay-dependent sufficient conditions for the ex- 


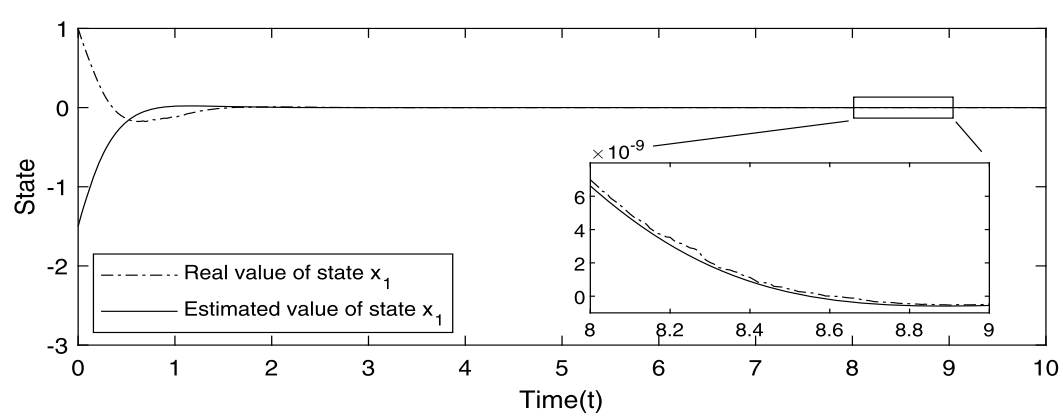

Figure 5 The simulation for state $x_{1}$ of the closed-loop systems with uncertainties

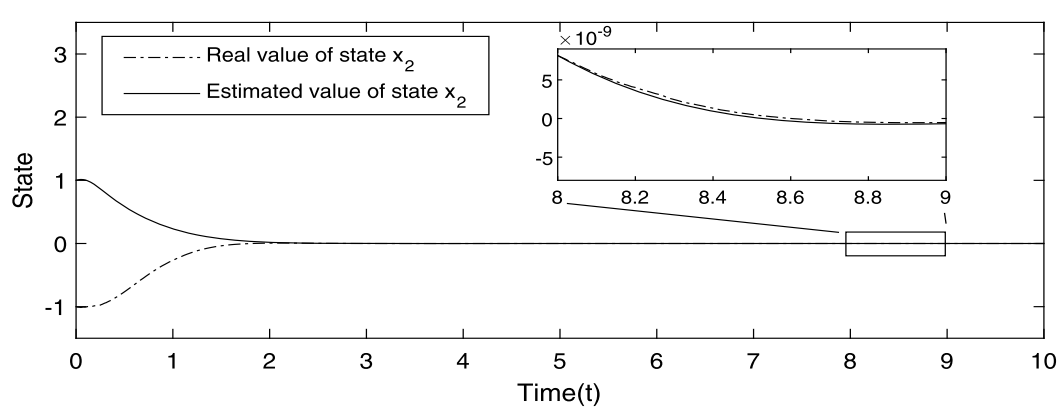

Figure 6 The simulation for state $x_{2}$ of the closed-loop systems with uncertainties

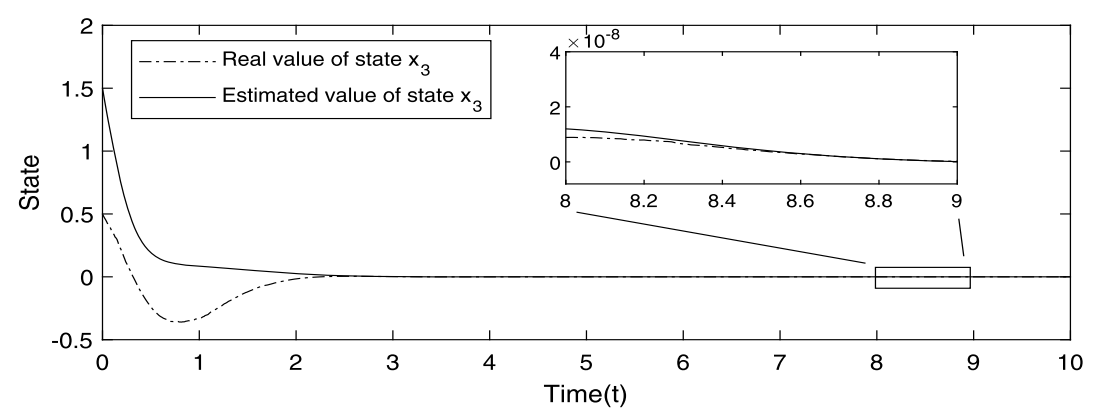

Figure 7 The simulation for state $x_{3}$ of the closed-loop systems with uncertainties

istence of the observer are given. Also, the desired gain matrix and state-feedback controller are constructed by solving certain LMIs, which can be implemented by using the LMI control toolbox. Moreover, one can readily obtain the delay-dependent results on the existence of an observer for the uncertain stochastic time-delay system. The numerical example has demonstrated the effectiveness of the proposed method.

Conflicts of interest

The authors declare that there is no conflict of interests regarding the publication of this article.

Funding

This research is supported by the Doctoral Research Fund of Harbin University of Commerce (2017BS011) and Young Innovative Talents Project of Harbin University of Commerce (2016QN047). 
Availability of data and materials

Data sharing not applicable to this article as no datasets were generated during the current study.

\section{Competing interests}

The authors declare that they have no competing interests.

\section{Authors' contributions}

All authors contributed equally to the writing of this paper. All authors read and approved the final manuscript.

\section{Author details}

${ }^{1}$ School of Basic Science, Harbin University of Commerce, Harbin, China. ${ }^{2}$ Department of Mathematics, Harbin Institute of Technology, Harbin, China.

\section{Publisher's Note}

Springer Nature remains neutral with regard to jurisdictional claims in published maps and institutional affiliations.

Received: 8 July 2019 Accepted: 29 October 2019 Published online: 14 November 2019

\section{References}

1. Xu, S.Y., Lam, J., Chen, T.W.: Robust $H_{\infty}$ control for uncertain discrete stochastic time-delay systems. Syst. Control Lett. $51,203-215(2004)$

2. Nowak, M., Sigmund, K.: The evolution of stochastic strategies in the prisoner's dilemma. Acta Appl. Math. 20, 247-265 (1990)

3. Mao, X.R., Marion, G., Renshaw, E.: Environmental noise suppresses explosion in population dynamics. Stoch. Process. Appl. 97, 95-110 (2002)

4. Wan, L., Zhou, Q.H.: Stochastic Lotka-Volterra model with infinite delay. Stat. Probab. Lett. 79, 698-706 (2009)

5. Niu, Y.G., Ho, D.W.C., Lam, J.: Robust integral sliding mode control for uncertain stochastic systems with time-varying delay. Automatica 41, 873-880 (2005)

6. Wang, J., Li, C.: Stabilization of a class of stochastic systems with time delays. J. Appl. Math. (2014) https://doi.org/10.1155/2014/274681

7. Malek-Zavarei, M., Jamshidi, M.: Time Delay Systems: Analysis, Optimization and Applications. North Holland, Amsterdam (1987)

8. Luan, X., Liu, F., Shi, P.: Finite-time filtering for non-linear stochastic systems with partially known transition jump rates. IET Control Theory Appl. 45, 735-745 (2009)

9. He, Y., Wang, Q.G., Lin, C., Wu, M.: Delay-range-dependent stability for systems with time-varying delay. Automatica 43(2), 371-376 (2007)

10. Chen, L., Han, Z., Ma, Z.: Exponential stability for impulsive stochastic nonlinear network systems with time delay. J. Appl. Math. (2014) https://doi.org/10.1155/2014/787568

11. Sakthivel, R., Mathiyalagan, K., Marshal Anthoni, S.: Robust stability and control for uncertain neutral time delay systems. Int. J. Control 85, 373-383 (2012)

12. Lu, R., Li, H., Zhu, Y: Quantized $H_{\infty}$ filtering for singular time-varying delay systems with unreliable communication channel. Circuits Syst. Signal Process. 31(2), 521-538 (2012)

13. Sun, J., Liu, G.P., Chen, J., Rees, D.: Improved delay-range-dependent stability criteria for linear systems with time-varying delays. Automatica 46(2), 466-470 (2010)

14. Zhang, H., Liu, Z., Huang, G.B.: Novel delay-dependent robust stability analysis for switched neutral-type neural networks with time-varying delays via SC technique. IEEE Trans. Syst. Man Cybern., Part B, Cybern. 40(6), 1480-1491 (2010)

15. Zheng, C.D., Shan, Q.H., Zhang, H., Wang, Z.: On stabilization of stochastic Cohen-Grossberg neural networks with mode-dependent mixed time-delays and Markovian switching. IEEE Trans. Neural Netw. Learn. Syst. 24(5), 800-811 (2013)

16. Feng, Z.G., Lam, J., Gao, H.J.: $\alpha$-Dissipativity analysis and synthesis of singular time-delay systems. Automatica 47 , 2548-2552 (2011)

17. Feng, Z.G., Lam, J., Yang, G.H.: Optimal partitioning method for stability analysis of continuous/discrete delay systems. Int. J. Robust Nonlinear Control (2013). https://doi.org/10.1002/rnc.3106

18. Park, P: A delay-dependent stability criterion for systems with uncertain time-invariant delays. IEEE Trans. Autom. Control 44, 876-877 (1999)

19. Moon, Y.S., Park, P., Kwon, W.H., Lee, Y.S.: Delay-dependent robust stabilization of uncertain state-delayed systems. Int. J. Control 74, 1447-1455 (2001)

20. Wang, Z.D., Huang, B., Unbehauen, H.: Robust $H_{\infty}$ observer design of linear time-delay systems with parametric uncertainty. Syst. Control Lett. 42, 303-312 (2001)

21. Tong, S.C., Li, C.Y., Li, Y.M.: Fuzzy adaptive observer backstepping control for MIMO nonlinear systems. Fuzzy Sets Syst. $160(19), 2755-2775(2009)$

22. Liu, Y.G., Zhang, J.F.: Reduced-order observer-based control design for nonlinear stochastic systems. Syst. Control Lett. $52,123-135(2004)$

23. Rathinasamy, S., Karimi, H.R., Raajananthini, K., et al.: Observer-based tracking control for switched stochastic systems based on a hybrid 2-D model. Int. J. Robust Nonlinear Control 28(2), 478-491 (2018)

24. Sakthivel, R., Susana Ramya, L., Selvaraj, P.: Observer-based state tracking control of uncertain stochastic systems via repetitive controller. Int. J. Control 48, 2272-2281 (2017)

25. Ma, H.J., Yang, G.H.: Filtering for a class of nonlinear MIMO uncertain time-delay stochastic systems. Int. J. Control $83(2), 432-440(2010)$ 
26. Jung, J., Huh, K., Lee, T.H.: Observer design methodology for stochastic and deterministic robustness. Int. J. Control 81(7), 1172-1192 (2008)

27. Niu, Y.G., Ho, D.W.C.: Robust observer design for Itô stochastic time-delay systems via sliding mode control. Syst. Control Lett. 55, 781-793 (2006)

28. Wang, C., Shen, Y.: Delay-range-dependent stability for uncertain stochastic systems with interval time-varying delay and nonlinear perturbations. Int. J. Comput. Math. 89(8), 983-997 (2012)

29. Boyd, S., Ghaoui, L., Feron, E., et al.: Linear Matrix Inequalities in System and Control Theory. Studies in Applied Mathematics. SIAM, Philadelphia (1994)

30. Raja, R., Anthoni, S.M.: Stability criteria for stochastic recurrent neural networks with two time-varying delays and impulses. Int. J. Comput. Appl. 1(28), 28-35 (2010)

31. Luenberger, D.G.: Observers for multi-variable systems. IEEE Trans. Autom. Control 11, 190-197 (1966)

32. Mao, X.R.: Stochastic Differential Equations and Applications. Horwood, Chichester (1997)

33. Xie, L., Khargonekar, P.P.: Lyapunov-based adaptive state estimation for a class of nonlinear stochastic systems. Automatica 48, 1423-1431 (2012)

34. Chen, M., Chen, W.H.: Disturbance-observer-based robust control for time delay uncertain systems. Int. J. Control. Autom. Syst. 8(2), 445-453 (2010)

35. Higham, D.: An algorithmic introduction to numerical simulation of stochastic differential equations. SIAM Rev. 43, 525-546 (2001)

\section{Submit your manuscript to a SpringerOpen ${ }^{0}$ journal and benefit from:}

- Convenient online submission

- Rigorous peer review

- Open access: articles freely available online

- High visibility within the field

- Retaining the copyright to your article

Submit your next manuscript at $\boldsymbol{~ s p r i n g e r o p e n . c o m ~}$ 